\title{
Survey papers in multimedia - guest editorial
}

\author{
Ramesh Jain • Albert Del Bimbo • Tat-Seng Chua • \\ Borko Furht
}

Published online: 29 December 2010

(C) Springer Science+Business Media, LLC 2010

\section{Guest Editorial}

Multimedia field has dramatically grown in the last 20 years. We still remember the first ACM Multimedia Conference in Anaheim, CA in 1993, at which most participants even did not know the meaning of the word "multimedia". In its early age, research in multimedia was focused on video and image compression and developing relevant standards, multimedia communication and synchronization, storage and retrieval, multimedia modeling, and creating first multimedia applications. Today, multimedia is interdisciplinary field, which is in tremendous explosion finding applications in practically all parts of our lives, from art, education, advertisement, and entertainment to engineering, medicine, business, scientific research, and many others.

We created two special issues:

Survey Papers in Multimedia by World Experts and Hot Research Topics in Multimedia

We invited experts in the field both from academia and industry to contribute with their articles, and the response was overwhelming. This special issue "Survey Papers" contains 13 articles presenting the state of the art in the various areas of multimedia, from context modeling, semantic retrieval, personalized multimedia, to multimedia data mining, augmented reality, and others. The main objective of this special issue is to present the current status of multimedia field from leading researchers and practitioners worldwide.

We thank all authors for their contributions in creating these two special issues.

Guest Editors

Ramesh Jain, Albert Del Bimbo, Tat-Seng Chua, and Borko Furht

R. Jain

Information \& Computer Sciences, University of California, Irvine, CA, USA

A. Del Bimbo

University of Florence, Florence, Italy

T.-S. Chua

School of Computing, National University of Singapore (NUS), Kent Ridge, Singapore

B. Furht $(\bowtie)$

Department of Computer \& Electrical Engineering and Computer Science, Florida Atlantic University, Boca Raton, FL, USA

e-mail: bfurht@fau.edu 


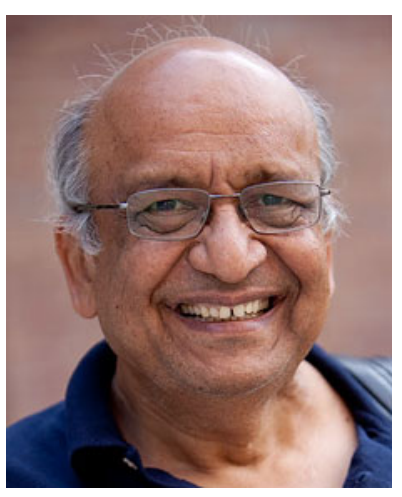

Ramesh Jain is an educator, researcher, and entrepreneur. Currently he is a Donald Bren Professor in Information \& Computer Sciences at University of California, Irvine. Earlier he served on faculty of Georgia Tech, University of California at San Diego, The university of Michigan, Ann Arbor, Wayne State University, and Indian Institute of Technology, Kharagpur. He is a Fellow of ACM, IEEE, AAAI, IAPR, and SPIE. His current research interests are in searching multimedia data and creating EventWebs for experiential computing.

Ramesh co-founded four companies, managed them in initial stages, and then turned them over to professional management. These companies were PRAJA in event-based business activity monitoring (acquired by Tibco); Virage for media management solutions and visual information management ?(a NASDAQ company acquired by Autonomy); and ImageWare for surface modeling, reverse engineering rapid prototyping, and inspection (acquired by SDRC). Currently, he serves as advisor to four companies and is a co-founder of mChron.

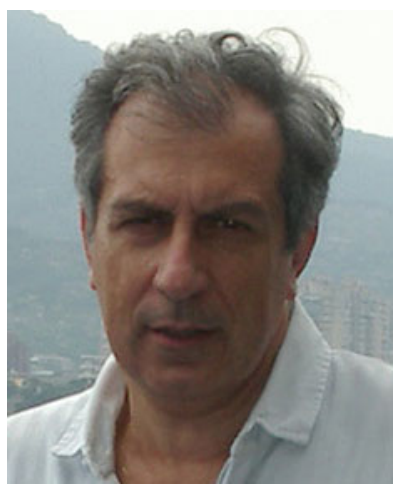

Alberto Del Bimbo is Full Professor of Computer Engineering, and Director of the Media Integration and Communication Center and Director of the Master in Multimedia at the University of Florence. He was the Deputy Rector for Research and Innovation Transfer of the University of Florence from 2000 to 2006 and the President of the Foundation for Research and Innovation from 2007 to 2009. His scientific interests are Multimedia Information Retrieval, Image and Video Analysis and Pattern Recognition, and Natural Human Computer Interaction. He has published over 250 publications in some of the most distinguished scientific journals and international conferences, and is the author of the monography "Visual Information Retrieval". From 1996 to 2000, he was the President of the IAPR Italian Chapter, and, from 1998 to 2000, Member at Large of the IEEE Publication Board. He was the general Chair of IAPR ICIAP'97, the International Conference on Image Analysis and Processing, IEEE ICMCS'99, the International Conference on Multimedia Computing and Systems, Program Co-Chair of ACM Multimedia 2008, and the General CoChair of ACM Multimedia 2010. He is the General Co-Chair of ICMR 2011, the International Conference on Multimedia Retrieval and of ECCV 2012, the European Conference on Computer Vision, and Program Co-Chair of ICPR 2012. He is IAPR Fellow and Associate Editor of Multimedia Tools and Applications, Pattern Analysis 
and Applications, Journal of Visual Languages and Computing and International Journal of Image and Video Processing, and was Associate Editor of Pattern Recognition, IEEE Transactions on Multimedia and IEEE Transactions on Pattern Analysis and Machine Intelligence.

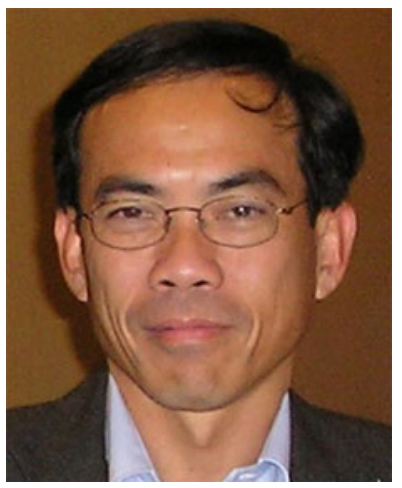

Chua Tat-Seng the KITHC Chair Professor at the School of Computing, National University of Singapore (NUS). He was the Acting and Founding Dean of the School of Computing during 1998-2000. He joined NUS in 1983, and spent three years as a research staff member at the Institute of Systems Science (now $I^{2} R$ ) in the late 1980s. Dr Chua's main research interest is in multimedia information retrieval, in particular, on the analysis, retrieval and question-answering (QA) of text and image/video information. He is currently working on several multi-million-dollar projects: interactive media search, local contextual search, and real-time live media search. His group participates regularly in TREC-QA and TRECVID video retrieval evaluations.

Dr Chua has organized and served as program committee member of numerous international conferences in the areas of computer graphics, multimedia and text processing. He is the conference co-chair of ACM Multimedia 2005, CIVR (Conference on Image and Video Retrieval) 2005, and ACM SIGIR 2008. He will serve as Technical PC Co-Chair of SIGIR 2011. He serves in the editorial boards of: ACM Transactions of Information Systems (ACM), Foundation and Trends in Information Retrieval (NOW), The Visual Computer (Springer Verlag), and Multimedia Tools and Applications (Kluwer). He is the member of steering committee of ACM ICMR (Int'1 Conference on Multimedia Retrieval), Computer Graphics International, and Multimedia Modeling conference series; and as member of International Review Panels of two large-scale research projects in Europe.

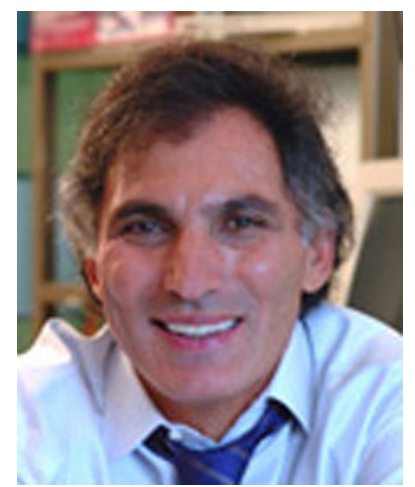

Borko Furht is a professor and chairman of the Department of Computer Science and Engineering at Florida Atlantic University in Boca Raton. He is also Director of the NSF-sponsored Industry/University Cooperative Research Center at FAU. His current research is in multimedia systems, video coding and compression, 3D video and image systems, video databases, wireless multimedia, and Internet and cloud computing. He has been Principal Investigator and Co-PI of several multiyear, multimillion dollar projects including the Center for Coastline Security Technologies, funded by the Department of Navy, One Pass to Production, funded by Motorola, the NSF PIRE project on Global Living Laboratory for Cyber Infrastructure Application Enablement, 
and the NSF funded High-Performance Computing Center. He is the author of numerous books and articles in the areas of multimedia, computer architecture, real-time computing, and operating systems. He is a founder and editor-in-chief of the Journal of Multimedia Tools and Applications (Springer, 1993). His latest books include "Handbook of Multimedia for Digital Entertainment and Arts" (Springer, 2009) and "Handbook of Media Broadcasting" (CRC Press, 2008). He has received several technical and publishing awards, and has consulted for many high-tech companies including IBM, Hewlett-Packard, Xerox, General Electric, JPL, NASA, Honeywell, and RCA, and has been an expert wetness for Cisco and Qualcomm. He has also served as a consultant to various colleges and universities. He has given many invited talks, keynote lectures, seminars, and tutorials. He serves on the Board of Directors of several high-tech companies. 